Physica D 30 (1988) 343-362

North-Holland, Amsterdam

\title{
SINGULAR MANIFOLDS AND QUASI-PERIODIC SOLUTIONS OF HAMILTONIANS FOR VORTEX LATTICES
}

\author{
Chjan C. LIM \\ Mathematics Department, University of Michigan, Ann Arbor, MI 48109, USA
}

Received 3 May 1987

Revised manuscript received 2 December 1987

\begin{abstract}
A general method for establishing the existence of quasi-periodic solutions of Hamiltonian systems for vortex lattices is illustrated in a simple example involving two degrees of freedom. The geometry of intersecting singular manifolds of the Hamiltonians introduces suitable canonical transformations which put the Hamiltonian into the form of singular weakly coupled oscillators. As by-products of this procedure, additional integrals of motion are found for the leading term in the transformed Hamiltonian. These extra integrals are approximate invariants for the full Hamiltonians.
\end{abstract}

\section{Introduction}

In this paper, a method for proving the existence of quasi-periodic solutions in finite-dimensional Hamiltonian systems is introduced and applied to a family of Hamiltonians (2.2) from vortex trail dynamics. Although it can be used in more general situations, we describe the method in the nontrivial context of Hamiltonians of two degrees of freedom, (2.6).

Meyer [1] discussed a method for finding periodic solutions in $N$-body problems which required the introduction of small parameters in judicious ways. Amongst the extensive literature on Hamiltonian mechanics, we refer the reader to some key references on weakly coupled oscillators of two degrees of freedom, i.e. Braun [2], Kummer [3, 4], Churchill et al. [5], Gustavson [6], Greene [7], Contopoulos [8]. In these papers, approximate third integrals and the existence of quasi-periodic solutions for resonantly coupled oscillators are discussed. The Hamiltonians considered in these papers have an isolated equilibrium at the origin.

On the other hand, the Hamiltonians (2.6) in this paper can be transformed into singular coupled oscillators, i.e. the origin is a branch point singularity of the Hamiltonian function of two complex variables. We will show that Hamiltonians with complicated singularities which lie on submanifolds are commonly found in vortex trail dynamics. The main idea in our method is to characterize the singularities of the Hamiltonian explicitly as embedded submanifolds in phase space and then use the natural coordinates of these singular manifolds to factor the Hamiltonian into the form of weakly coupled singular oscillators in the neighborhood of points where two or more singular manifolds intersect. Once this has been carried out, an approximate third integral of eqs. (A.1), of which (2.6) is the reduced Hamiltonian, can be found.

We consider a class of Hamiltonians which govern the nonlinear dynamics of infinite lattices of point vortices in two-dimensional inviscid flows. Of special interest to us are the one-dimensional lattices which model the wake behind a cylinder and the plane jet. They are known as the von Karman trail and the symmetric trail respectively (see fig. 1). von Karman [9] discussed the linear stability of such objects under 
periodic disturbances. Sirovich [10] used the linear oscillation theory of the von Karman trail to describe quasi-periodic flows behind the cylinder (see Sreenivasan [11]). Lim and Sirovich [12a] discussed linear wave propagation theory while Lim and Sirovich [12b] studied the linear stability of the von Karman trail to aperiodic disturbances of compact support. Kochin [13] was the first author to discuss the nonlinear dynamics of such vortex trails. Lim and Sirovich [14] discussed numerical solutions of the vortex trail far from equilibrium configurations, which correspond to vortex merging in the wake of a cylinder. One of the chief goals here is to prove the existence of quasi-periodic solutions and open connected sets of bounded solutions for these Hamiltonians, (2.6).

A related but different problem in vortex dynamics is the integrability of Hamiltonians for $N$ point vortices. Early work on this problem can be found in Synge [15] and Lin [16]. The case of $N=3$ was found to be completely integrable. (See Novikov [17] and Aref [18].) For $N=4$, Novikov [19] showed numerically that chaotic solutions exist. On the other hand, Khanin [20] proved that some quasi-periodic solutions exist for this case.

We summarize the main steps in our approach:

(i) derivation of finite-dimensional Hamiltonians from periodic problems for the infinite lattices, e.g. the vortex trails;

(ii) classification of the complex singularities of the Hamiltonians, i.e. branch points and describe their geometry as singular manifolds;

(iii) factorization of Hamiltonians near intersections of singular manifolds to obtain weakly coupled singular oscillators;

(iv) establish the existence of quasi-periodic solutions by KAM theory;

(v) establish the existence of open connected subsets of bounded solutions.

In section 2, we briefly review step (i) above. We discuss the singularities in section 3 . One class of quasi-periodic solutions is considered in section 4 and a different class in section 5 . In the conclusion, section 7 , we discuss the connection between our theoretical results and experiments.

\section{Reduced Hamiltonians}

Here we briefly review the derivation of finite-dimensional Hamiltonians for the periodic problem of the vortex trails (see Kochin [13]).

An $N$-periodic disturbance of a vortex trail can be viewed in terms of repeated groups of $2 N$ vortices. The corresponding vortices in each group form an infinite vortex row. This idea is depicted for the case $N=2$ in fig. 1 . Thus the evolution of periodic disturbances on vortex trails can be formated in terms of equations describing the interaction of parallel vortex rows.

The complex velocity potential for the $j$ th row is given by

$$
W_{j}(Z)=\frac{(-1)^{j} \Gamma}{2 \pi i} \log \sin \left[\frac{\pi}{N l}\left(Z-Z_{j}\right)\right]
$$

where $Z_{j}$ is the location of a typical vortex in the $j$ th row and $l$ is the distance between adjacent vortices in the original vortex trail. The factor $(-1)^{j}$ adjusts the circulation so that odd-numbered rows have circulation $-\Gamma$ and even-numbered rows have circulation $\Gamma$. Using (2.1), the equations for the $N$-periodic 


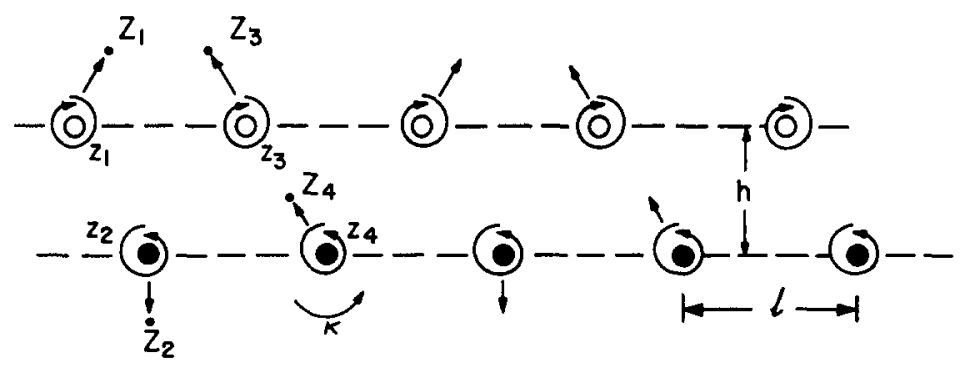

Fig. 1a. The von Karman trail consists of two rows of point vortices of opposite circulations denoted by open and filled circles. The distance between two vortices in each row is denoted by $l$. The separation between rows is denoted by $h$. $b$ denotes the stagger between the two rows; in this case $b=l / 2$.

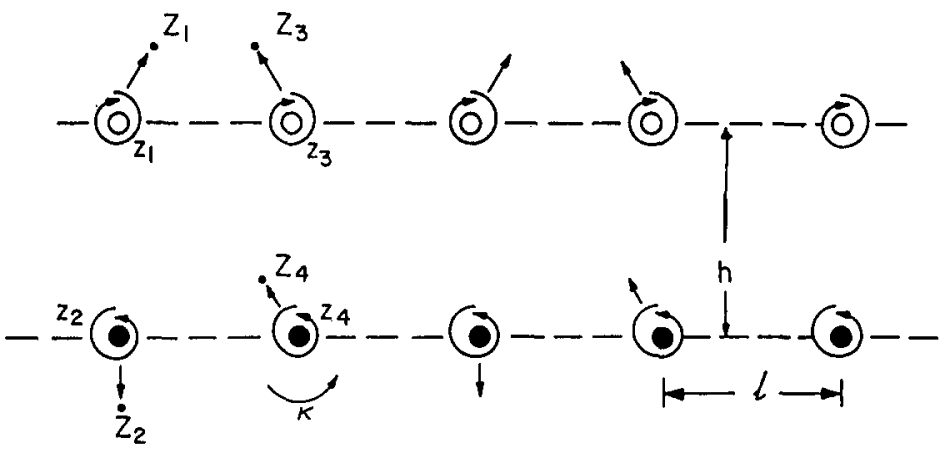

Fig. 1b. The symmetric trail corresponds to the case where the stagger $b=0$. The arrows in both figures describe the period two $(N=2)$ perturbation of the equilibrium trails. The vortices $\left\{Z_{1}, Z_{2}, Z_{3}, Z_{4}\right\}$ in both figures form a 4 -cell. $\kappa$ denotes the circulation strength of the vortices.

problem (or $2 N$ cell problem) can be written in the form

$$
\frac{\mathrm{d} \bar{Z}_{k}}{\mathrm{~d} t}=\sum_{j=1}^{2 N}(-1)^{j} \frac{\Gamma}{2 N l i} \cot \frac{\pi}{N l}\left(Z_{k}-Z_{j}\right), \quad k=1, \ldots, 2 N,
$$

where prime denotes exclusion of the term $j=k$ in the sum, and bar denotes complex conjugation. This is a Hamiltonian system with $N$ degrees of freedom.

Note that (2.2) has the integral

$$
C=\sum_{i \text { odd }} Z_{i}-\sum_{j \text { even }} Z_{j}
$$

which is the second integral for (2.2). The integral manifolds of (2.2) have a trivial noncompactness property which can be removed by using the translational invariance of the equations. This implies that (2.2) can be transformed into $(2 N-1)$ complex equations in terms of the differences. We will focus on the $N=2$ case which is the lowest-dimensional problem of importance in vortex trail dynamics. The transformed equations in terms of the three differences $\left(Z_{1}-Z_{2}\right),\left(Z_{2}-Z_{3}\right)$ and $\left(Z_{3}-Z_{4}\right)$ are given in 
the appendix, (A.1). The integral (2.3) takes the form

$$
C=\left(Z_{1}-Z_{2}\right)+\left(Z_{3}-Z_{4}\right)
$$

for the case $N=2$. The $C$-level sets are thus invariant manifolds and we consider the reduced flow on these manifolds.

Instead of writing the equations for the reduced flow in terms of the above differences, e.g. $\left(Z_{1}-Z_{2}\right)$ etc., we follow Kochin [13] and give the equations in terms of non-dimensionalized perturbations from the corresponding equilibria. This is sketched in the appendix. The flow on the $C$-level manifolds are thus governed by the equations

$$
\begin{aligned}
& \frac{\mathrm{d} \bar{\alpha}}{\mathrm{d} \tau}=-\mathrm{i} \sin \beta\left[\frac{1}{\cos \alpha+\cos \beta}-\frac{1}{\cos \beta+\cos G}\right], \\
& \frac{\mathrm{d} \bar{\beta}}{\mathrm{d} \tau}=-\mathrm{i} \sin \alpha\left[\frac{1}{\cos \alpha+\cos \beta}-\frac{1}{\cos \alpha-\cos G}\right] .
\end{aligned}
$$

The associated complex Hamiltonian parametrized by $G$ is the following function of two complex variables $(\alpha, \beta)$ :

$$
H=\log \left\{\frac{(\cos \alpha-\cos G)(\cos \beta+\cos G)}{\cos \alpha+\cos \beta}\right\}
$$

The complex equations (2.5) are then given by

$$
\frac{\mathrm{d} \bar{\alpha}}{\mathrm{d} \tau}=\mathrm{i} \frac{\partial H}{\partial \beta}, \quad \frac{\mathrm{d} \bar{\beta}}{\mathrm{d} \tau}=\mathrm{i} \frac{\partial H}{\partial \alpha},
$$

where the partial derivatives in (2.7) are taken in the sense of the theory of several complex variables. These equations are not in the standard Hamiltonian form but are convenient. In standard form eqs. (2.7) become

$$
\begin{aligned}
& \frac{\mathrm{d} \alpha_{\mathrm{r}}}{\mathrm{d} \tau}=\frac{\partial H}{\partial \beta_{\mathrm{i}}}, \quad \frac{\mathrm{d} \beta_{\mathrm{i}}}{\mathrm{d} \tau}=-\frac{\partial H}{\partial \alpha_{\mathrm{r}}} \\
& \frac{\mathrm{d} \beta_{\mathrm{r}}}{\mathrm{d} \tau}=\frac{\partial H}{\partial \alpha_{\mathrm{i}}}, \quad \frac{\mathrm{d} \alpha_{\mathrm{i}}}{\mathrm{d} \tau}=-\frac{\partial H}{\partial \beta_{\mathrm{r}}}, \\
& H=\log \left|\frac{(\cos \alpha-\cos G)(\cos \beta+\cos G)}{\cos \alpha+\cos \beta}\right|,
\end{aligned}
$$

where the absolute value sign denotes the modulus in complex function theory and $\alpha=\alpha_{\mathrm{r}}+\mathrm{i} \alpha_{\mathrm{i}}$, $\beta=\beta_{\mathrm{r}}+\mathrm{i} \beta_{\mathrm{i}}$. Henceforth we will use the complex form of the equations. Observe that the complex-valued parameter

$$
G=\frac{\pi}{2 l}\left[Z_{10}-Z_{20}+Z_{30}-Z_{40}\right]
$$

is essentially the non-dimensionalized value of the integral $C(2.4)$. 
We summarize the above considerations in the following:

Theorem 2.1. The reduced flow on the $C$-level manifolds of (A.1) is given by the 2-parameter family of Hamiltonians $H(\alpha, \beta ; G),(2.6)$, which depends continuously on the complex parameter $G$.

\section{Singularities}

The Hamiltonian $H(\alpha, \beta ; G),(2.6)$, is an analytic function of two complex variables $\alpha, \beta$ except at the singularities of the logarithm function. The branch cut of the log function does not play a role here because for given values of $(\alpha, \beta)$, one can choose the branch cut to avoid the value of the argument of the $\log$ function. Furthermore the complex derivative of the $\log$ function is the same for all branches. On the other hand, the branch point of the log function is important for our analysis.

To elaborate on the structure of these singularities we write the Hamiltonian (2.6) in the form

$$
H(\alpha, \beta ; G)=-\log (\cos \alpha+\cos \beta)+\log (\cos \alpha-\cos G)+\log (\cos \beta+\cos G)
$$

The set of singularities denoted by $\Omega(G)$ consists of values of $(\alpha, \beta)$ which give branch points of at least one of the three $\log$ functions in (3.1). Since the arguments of the $\log$ functions in (3.1) are functions of two complex variables, their zeros are in general complex hypersurfaces in $(\alpha, \beta)$ phase space. Thus the singular set consists of the union

$$
\begin{aligned}
\Omega(G)= & \{(\alpha, \beta) \mid(\cos \alpha-\cos G)=0\} \cup\{(\alpha, \beta) \mid(\cos \beta+\cos G)=0\} \\
& \cup\{(\alpha, \beta) \mid(\cos \alpha+\cos \beta)=0\} .
\end{aligned}
$$

On $\Omega(G)$, the vector field (2.6) is undefined, i.e. blows up.

The entire functions in (3.2) have multi-valued inverses. Thus

$$
\Omega(G)=\{(\alpha, \beta) \mid \alpha= \pm G, \beta \in \mathbb{C}\} \cup\left\{(\alpha, \beta) \mid \beta_{\mathrm{r}}=G_{\mathrm{r}}+\pi, \beta_{\mathrm{i}}=G_{\mathrm{i}}, \alpha \in \mathbb{C}\right\} \cup\{(\alpha, \beta) \mid \alpha \pm \beta=\pi\}
$$

Note that the first two subsets are parametrized smoothly by $G$ while the third and fourth sets do not depend on $G$.

Next, we give coordinates which are natural to the singular manifolds comprising $\Omega(G)$, i.e. charts for these manifolds. First we define the singular points

$$
O_{1}=(\alpha=G, \beta=G-\pi), \quad O_{2}=(\alpha=-G, \beta=G+\pi),
$$

which serve as the origins of two coordinate systems

$$
\left(a^{\prime}, b^{\prime}\right)=(\alpha, \beta)-O_{1}, \quad(a, b)=(\alpha, \beta)-O_{2}
$$

It is important to note that the first subset of $\Omega(G)$ consists of the 2-submanifolds

$$
\begin{aligned}
& \mu_{1}=\left\{\left(a^{\prime}, b^{\prime}\right) \mid a^{\prime}=0, b^{\prime} \in \mathbb{C}\right\} \\
& \mu_{2}=\{(a, b) \mid a=0, b \in \mathbb{C}\}
\end{aligned}
$$


The second subset of $\Omega(G)$ consists of the 2-submanifolds

$$
\begin{aligned}
& \nu_{1}=\left\{\left(a^{\prime}, b^{\prime}\right) \mid a^{\prime} \in \mathbb{C}, b^{\prime}=0\right\}, \\
& \nu_{2}=\{(a, b) \mid a \in \mathbb{C}, b=0\} .
\end{aligned}
$$

All three subsets in (3.3) can be extended $\bmod (2 \pi)$ to countably many 2 -manifolds due to the inherent periodicity of the vortex trails considered (see fig. 1). The third subset of $\Omega(G)$ consists of the 2-manifolds

$$
\begin{aligned}
& \omega_{1}=\left\{\left(a^{\prime}, b^{\prime}\right) \mid a^{\prime}+b^{\prime} \in \mathbb{C}, a^{\prime}-b^{\prime}=0\right\}, \\
& \omega_{2}=\{(a, b) \mid a+b=0, a-b \in \mathbb{C}\} .
\end{aligned}
$$

The points $O_{i}$ which define the coordinate systems (3.5) are in fact the unique intersection points of the manifolds, i.e.

$$
O_{i}=\mu_{i} \cap \nu_{i} \cap \omega_{i}, \quad i=1,2 .
$$

We give a further coordinate transformation which also provides the trivial embedding for the manifolds $\omega_{i}$,

$$
\begin{aligned}
& A^{\prime}\left(a^{\prime}, b^{\prime}\right)=a^{\prime}+b^{\prime}, \quad B^{\prime}\left(a^{\prime}, b^{\prime}\right)=a^{\prime}-b^{\prime}, \\
& A(a, b)=a+b, \quad B(a, b)=a-b .
\end{aligned}
$$

In these coordinates

$$
\begin{aligned}
& \omega_{1}=\left\{\left(A^{\prime}, B^{\prime}\right) \mid A^{\prime} \in \mathbb{C}, B^{\prime}=0\right\}, \\
& \omega_{2}=\{(A, B) \mid A=0, B \in \mathbb{C}\} .
\end{aligned}
$$

Besides $O_{i}, i=1,2$, we will be concerned with a third intersection point, namely

$$
O_{3}=\omega_{1} \cap \omega_{2}=(\pi, 0) \text { in }(\alpha, \beta) \text { coordinates. }
$$

In small neighborhoods of the intersection points $O_{i}, i=1,2,3$, we prove the existence of quasi-periodic solutions of (2.6). The coordinates natural to the singular manifolds (3.5) and (3.10) will be used to factor the Hamiltonian (2.6) into weakly coupled singular oscillators, i.e. nearly integrable systems.

We summarize the above considerations on the topology of singular manifolds for (2.6):

\section{Theorem 3.1.}

(i) $\Omega(G)$ consists of the 2-submanifolds $\mu_{i}, \nu_{i}, \omega_{i}, i=1,2, \bmod 2 \pi$,

(ii) $\mu_{i}, \nu_{i}, \omega_{i}$ are complex hyperplanes in $\mathbb{C}^{2}$, and

(iii) $\mu_{i}, \nu_{i}, \omega_{i}$ intersect transversally at $O_{i}, i=1,2 ; \omega_{1}$ and $\omega_{2}$ intersect transversally at $O_{3}$.

As remarked earlier, on $\Omega(G)$ the $\log$ function in the Hamiltonian (2.6) blows-up. In fact it is easy to verify that

\section{Lemma 3.2.}

(i) $\operatorname{Re}(H)$ tends to $-\infty$ on $\mu_{i}, v_{i}, i=1,2$,

(ii) $\operatorname{Re}(H)$ tends to $+\infty$ on $\omega_{i}, i=1,2$. 
An immediate corollary of lemma 3.2 is

Theorem 3.3.

(i) $\mu_{i}, \nu_{i}, \omega_{i}$ are not integral manifolds,

(ii) trajectories of the Hamiltonian system (2.5) do not intersect these singular manifolds.

\subsection{Vortex merging}

Here we give the physical interpretations for the phase points on or near the singular manifolds. The transformations (3.5) and (3.10) are first inverted and then the definition of the variables $(\alpha, \beta)$ in the appendix is used to describe the singular manifolds in terms of the locations of the four vortex rows in the complex plane.

$\left\{\mu_{i}, \nu_{i}, i=1,2\right\}$ correspond to the merging of two vortex rows of opposite circulation while the remaining two rows are separate.

$\left\{\omega_{i}, i=1,2\right\}$ correspond to the merging of two vortex rows of similar circulation while the remaining two rows are separate.

$\left\{O_{i}, i=1,2\right\}$ correspond to the merging of three vortex rows with the fourth row remaining separate from the cluster of three.

$\left\{\mathrm{O}_{3}\right\}$ correspond to two concurrent mergings of similar vortex rows.

The following definitions will facilitate our discussion of vortex states which correspond to phase points in the neighborhoods of $\mu_{i}, \nu_{i}, \omega_{i}$.

\section{Definitions.}

(i) A vortex pair is two vortex rows of the same circulation relatively close together.

(ii) A vortex couple is two vortex rows of opposite circulation relatively close together.

(iii) A vortex triplet is three vortex rows relatively close together.

In this paper, we are concerned with bounded solutions of (2.6) and hence we do not discuss vortex couples which correspond to unbounded motions (see Lim and Sirovich [14]). We end this section with the remark that theorem 3.3 implies that vortex collapse cannot occur in the dynamics of four infinite vortex rows with equal and opposite circulations. In this respect, the dynamics of vortex rows differ from the problem of the motion of a finite number of point vortices. Novikov [17] showed that three vortices can collapse.

\section{Quasi-periodic solutions I}

We use the coordinate transformations (3.5) and (3.10) to write the Hamiltonian (2.6) in the form of weakly coupled singular oscillators of two degrees of freedom. A by-product of this factorization procedure is an approximate third integral for eqs. (A.1). Furthermore, the singular (and integrable) part of the transformed Hamiltonian turns out to be independent of the value of $G$ which parametrizes the reduced flow on the $C$-level manifolds of (A.1).

In the coordinates $(a, b)$ given by the second part of (3.5), the Hamiltonian (2.6) takes the form

$$
H(a, b ; G)=\log \left[\frac{a b}{a+b}\right]+\log [F(a, b ; G)],
$$


where

$$
F(a, b ; G)=1+\mathcal{O}(a, b)
$$

is a convergent power series in the complex variables $(a, b)$ starting with the term one, for $|a|,|b|$ sufficiently small.

The singular part takes the form

$$
H_{0}(a, b)=\log \left[\frac{a b}{a+b}\right]
$$

The Hamilton equations for $H_{0}$ are

$$
\begin{aligned}
& \frac{\mathrm{d} \bar{a}}{\mathrm{~d} \tau}=\mathrm{i} \frac{\partial H_{0}}{\partial b}=\frac{\mathrm{i} a}{(a+b) b}, \\
& \frac{\mathrm{d} \bar{b}}{\mathrm{~d} \tau}=\mathrm{i} \frac{\partial H_{0}}{\partial a}=\frac{\mathrm{i} b}{(a+b) a} .
\end{aligned}
$$

We carry out a second coordinate transformation given by (3.10). In these variables, and after rescaling time, eqs. (4.4) become

$$
\begin{aligned}
& \frac{\mathrm{d} \bar{A}}{\mathrm{~d} \tau}=\frac{\mathrm{i}}{A}\left[\frac{A^{2}+B^{2}}{A^{2}-B^{2}}\right]=\mathrm{i} \frac{\partial H_{0}}{\partial A}, \\
& \frac{\mathrm{d} \bar{B}}{\mathrm{~d} \tau}=2 \mathrm{i}\left[\frac{B}{A^{2}-B^{2}}\right]=\mathrm{i} \frac{\partial H_{0}}{\partial B},
\end{aligned}
$$

where the Hamiltonian is

$$
H_{0}(A, B)=\log \left[\frac{A^{2}-B^{2}}{A}\right]
$$

A second integral of (4.5) is given by

$$
I(A, B)=|A|^{2}-|B|^{2} .
$$

Further work is needed before we obtain the desired form of weakly coupled singular oscillators.

We pause here to summarize the results of the two coordinate transformations performed above.

\section{Theorem 4.1.}

(i) In a sufficiently small ball around the intersection of singular manifolds, $\mathrm{O}_{2}$ (3.4), the Hamiltonian (2.6) splits into a small perturbation of the singular part, i.e.

$$
H(A, B ; G)=H_{0}(A, B)+H_{1}(A, B ; G) .
$$

(ii) The singular part, $H_{0}(A, B)$ (4.6), gives rise to a completely integrable Hamiltonian system (4.5).

(iii) The level sets $I^{-1}(J), J \in \mathbb{R}$ are 3-hyperboloids in $\mathbb{R}^{4}$; the associated Lie group is $\mathrm{SU}(1,1)$. 
Proof.

(i) The term $H_{1}(A, B ; G)=\log [F(A, B ; G)]$, where $F(A, B ; G)=1+\mathcal{O}(A, B ; G)$ is a convergent power series in $A, B$ which begins with the term 1 and has coefficients which depend analytically on $G$. Thus in a sufficiently small ball around the origin, $(A, B)=(0,0), \log [F]$ is $\mathcal{O}(|A|,|B|)$. This proves part (i).

(ii) The second integral, $I=|A|^{2}-|B|^{2}$, is independent of the Hamiltonian $H_{0}(A, B)$. Thus (4.5) is completely integrable.

(iii) The manifolds, $I^{-1}(J), J \in \mathbb{R}$ are the noncompact 3-manifolds in $\mathbb{R}^{4}$ known as 3-hyperboloids. For $J=1$, the 3-hyperboloid is the group manifold for Lie group $\mathrm{SU}(1,1)$.

Using the primed coordinate systems (3.5) and (3.10) and carrying out exactly the same steps as above, we obtain a similar result for the intersection point $O_{1}$ (3.4). It is interesting that in the neighborhoods of intersection points of singular manifolds, a result such as theorem 4.1 can be established. The generalizations of such results to other classes of Hamiltonians is the subject of Lim [21].

\subsection{Singular decoupled oscillators}

Although it is possible to compute explicitly the quasi-periodic solutions for (4.5) (this is carried out in Lim and Sirovich [14]) and then continue these solutions to the full Hamiltonian (4.8), we follow another route where the conditions of KAM theory are easily verified. For this purpose, we construct open subsets of a small ball around the origin,

$$
S_{\varepsilon}=\left\{\left.(A, B)|| A\right|^{2}+|B|^{2}<\varepsilon\right\},
$$

in the following way:

$$
M_{\delta, n}=\left\{\left.(A, B) \in S_{\varepsilon}|\delta<| B\right|^{2}<\varepsilon-\delta^{2}, \frac{\delta^{2}}{n}<|A|^{2}<\delta^{2}\right\}
$$

where $\delta \ll \varepsilon(G)$ and $n$ is a large positive integer. These product domains of two annulus are chosen to be nearer to the singular manifold $\omega_{2}$ than to the origin of the coordinate system $(A, B)$. At the same time, they are bounded away from the singular manifolds. Thus the full Hamiltonian, $H(A, B)(4.8)$, is analytic in $M_{\delta, n}$.

Now we perform the second factorization on the Hamiltonian $H_{0}(A, B)(4.6)$ which splits it into three terms,

$$
H_{0}(A, B)=2 \log (B)-\log (A)+\log \left(1-A^{2} / B^{2}\right) .
$$

The third term $\log \left(1-A^{2} / B^{2}\right)$ is $\mathcal{O}\left(|A|^{2} /|B|^{2}\right)$ in the open sets $M_{\delta, n}$. Next we write the full Hamiltonian (4.8) as the sum of two terms,

$$
\begin{aligned}
& H(A, B)=H_{0}^{\prime}(A, B)+H_{1}^{\prime}(A, B), \\
& H_{0}^{\prime}(A, B)=2 \log (B)-\log (A), \\
& H_{1}^{\prime}(A, B ; G)=\log \left(1-A^{2} / B^{2}\right)+\log [F(A, B ; G)] .
\end{aligned}
$$

Note that we have collected together the small terms of $\mathcal{O}(\delta)$ and $\mathcal{O}(\varepsilon)$ respectively in the perturbation term $H_{1}^{\prime}(A, B ; G)$. 
Dropping primes, $H_{0}(A, B)$ in (4.12) is the Hamiltonian for decoupled oscillators. It is singular at $|A|=0$ or $|B|=0$. Thus we have achieved one of the main goals of this paper. The Hamiltonian equations for $H_{0}(A, B)(4.12)$ are

$$
\begin{aligned}
& \frac{\mathrm{d} \bar{A}}{\mathrm{~d} \tau}=-\frac{\mathrm{i}}{A}=\mathrm{i} \frac{\partial H_{0}}{\partial A}, \\
& \frac{\mathrm{d} \bar{B}}{\mathrm{~d} \tau}=-\frac{2 \mathrm{i}}{B}=-\mathrm{i} \frac{\partial H_{0}}{\partial B} .
\end{aligned}
$$

These equations are clearly nonlinear and therefore can be made non-resonant by appropriate choice of the moduli $|A|,|B|$.

Lemma 4.2. All orbits of (4.13) are in products of two circles, i.e. $S^{1} \times S^{1}=T^{2}$.

Proof. First we write $H_{0}(A, B)$ in the polar coordinates

$$
A=\sqrt{J_{1}} \mathrm{e}^{-\mathrm{i} \theta_{1}}, \quad B=\sqrt{J_{2}} \mathrm{e}^{\mathrm{i} \theta_{2}} .
$$

In action-angle variables, the real Hamiltonian is given by

$$
H_{0}\left(J_{1}, J_{2}\right)=2 \log \left(J_{2}\right)-\log \left(J_{1}\right) .
$$

Thus the complex form of Hamilton's equations (4.13) takes the real form

$$
\begin{aligned}
& \frac{\mathrm{d} J_{1}}{\mathrm{~d} \tau}=0, \quad \frac{\mathrm{d}}{\mathrm{d} \tau} \theta_{1}=-\frac{1}{J_{1}}=\frac{\partial H_{0}}{\partial J_{1}}, \\
& \frac{\mathrm{d} J_{2}}{\mathrm{~d} \tau}=0, \quad \frac{\mathrm{d}}{\mathrm{d} \tau} \theta_{2}=\frac{2}{J_{2}}=\frac{\partial H_{0}}{\partial J_{2}} .
\end{aligned}
$$

The orbits of these equations are clearly in $S^{1} \times S^{1}$.

Lemma 4.3. The frequencies of the decoupled oscillators (4.16) depend on the values of $J_{1}, J_{2}$. In particular, they can be rationally independent in which case the corresponding orbit is dense in $S^{1} \times S^{1}$.

Proof. The frequencies are given by

$$
f_{1}=-\frac{1}{J_{1}}=\frac{\partial H_{0}}{\partial J_{1}}, \quad f_{2}=\frac{2}{J_{2}}=\frac{\partial H_{0}}{\partial J_{2}} .
$$

Lemma 4.4. The Hamiltonian $H_{0}\left(J_{1}, J_{2}\right)$ is nondegenerate for all values of $J_{1}, J_{2}$.

Proof. The nondegeneracy condition of KAM theory is verified,

$$
\left|\begin{array}{l}
\frac{\partial^{2} H_{0}}{\partial J_{1}^{2}} \frac{\partial^{2} H_{0}}{\partial J_{1} \partial J_{2}} \\
\frac{\partial^{2} H_{0}}{\partial J_{2} \partial J_{1}} \frac{\partial^{2} H_{0}}{\partial J_{2}^{2}}
\end{array}\right|=\left|\begin{array}{cc}
\frac{1}{J_{1}^{2}} & 0 \\
0 & -\frac{2}{J_{2}^{2}}
\end{array}\right|=-\frac{2}{J_{1}^{2} J_{2}^{2}},
$$


for all values of $J_{1}, J_{2}>0$. In fact the determinant can be made arbitrarily large near the singular intersection point $\mathrm{O}_{2}$.

\subsection{Continuation to full Hamiltonian}

In order to continue the quasi-periodic solutions $S^{1} \times S^{1}$ of the leading Hamiltonian $H_{0}\left(J_{1}, J_{2}\right)$ to the full Hamiltonian we gather here the properties of $H\left(J_{1}, J_{2}, \theta_{1}, \theta_{2} ; G\right)$. This Hamiltonian is the sum of two terms of which $H_{1}$ is small in the sets $M_{\delta, n}$,

$$
\begin{aligned}
& H\left(J_{1}, J_{2}, \theta_{1}, \theta_{2} ; G\right)=H_{0}\left(J_{1}, J_{2}\right)+H_{1}\left(J_{1}, J_{2}, \theta_{1}, \theta_{2} ; G\right) \\
& H_{1}\left(J_{1}, J_{2}, \theta_{1}, \theta_{2} ; G\right)=\log \left\{1-J_{1} / J_{2} \cos 2\left(\theta_{1}+\theta_{2}\right)+J_{1}^{2} / J_{2}^{2}\right\}+\log \left\{F\left(J_{1}, J_{2}, \theta_{1}, \theta_{2} ; G\right)\right\}
\end{aligned}
$$

In the second term in $H_{1}$,

$$
F\left(J_{1}, J_{2}, \theta_{1}, \theta_{2} ; G\right)=|F(A, B ; G)|
$$

is the modulus of the $\mathcal{O}(1)$ second term in $H_{1}^{\prime}(A, B ; G)$ (4.12). Since $F(A, B ; G)$ is a convergent power series in $A, B$ with leading term equal to one, (4.20) is real analytic in $J_{1}, J_{2}, \theta_{1}, \theta_{2}$. It is $2 \pi$-periodic in $\theta_{1}, \theta_{2}$ because these angle variables enter (4.20) in the form of harmonics of the trigonometric functions $\cos \theta_{1}, \cos \theta_{2}, \sin \theta_{1}, \sin \theta_{2}$. Furthermore $F\left(J_{1}, J_{2}, \theta_{1}, \theta_{2} ; G\right)$ is $\mathcal{O}(1)$.

We are now ready to state and prove our main result.

Theorem 4.5. In the open sets $M_{\delta, n}$ for $\delta \ll \varepsilon(G) \ll 1$ and $n \in \mathbb{Z}^{+}$large, the full Hamiltonian (4.19) admits a set of quasi-periodic solutions which has positive Lebesgue measure in $\mathbb{R}^{4}$.

Proof. The Hamiltonian (4.19) is real analytic in $J_{1}, J_{2}, \theta_{1}, \theta_{2}$ in $M_{\delta, n}$ and $2 \pi$-periodic in $\theta_{1}, \theta_{2}$. The leading term $H_{0}(4.15)$ is independent of $\theta_{1}, \theta_{2}$ and admits quasi-periodic solutions $\mathrm{S}^{1} \times \mathrm{S}^{1}$ in $M_{\delta, n}$ (cf. lemmas $4.2,4.3)$. Lemma 4.4 states that $H_{0}\left(J_{1}, J_{2}\right)$ is nondegenerate for all values of $J_{1}, J_{2}$ in $M_{\delta, n}$.

In the open sets $M_{\delta, n}, J_{1} / J_{2}<\delta \ll \varepsilon$ and $J_{2}<\varepsilon(G)$ which implies that the perturbation term $H_{1}\left(J_{1}, J_{2}, \theta_{1}, \theta_{2} ; G\right)(4.19)$ is $\mathcal{O}(\varepsilon)$ in $M_{\delta, n}$. Thus all the conditions of the KAM theory (see Arnol'd [22, 23], Moser [24, 25]), are satisfied and this proves the theorem.

We end this section with the remark that theorem 4.5 holds for all values of $G$ because the full Hamiltonian (4.19) is smoothly parametrized by the complex parameter $G$ but the leading term $H_{0}\left(J_{1}, J_{2}\right)$ (4.5) is independent of $G$.

We remind the reader that (4.19) is the reduced Hamiltonian on the $C$-level manifolds of eqs. (A.1); the parameter $G$ specifies the $C$-level manifold.

In addition, it should be noted that an analogue of theorem 4.5 for the singular intersection point $O_{1}$ (3.4), (3.9) is proved in a similar way. These remarks imply the following theorem.

Theorem 4.6. On each $C$-level manifold of the equations for four vortex rows, (A.1), the reduced Hamiltonian (2.6) admits quasi-periodic solutions in open subsets $M_{\delta, n}$ and $M_{\delta, n}^{\prime}$ respectively of sufficiently small neighborhoods $S_{\varepsilon}$ and $S_{\varepsilon}^{\prime}$ of the singular intersection points $O_{2}, O_{1}$. 


\section{Quasi-periodic solutions II}

In this section, the existence of quasi-periodic solutions in a full neighborhood of the third singular intersection point $O_{3},(3.12)$ is established. We recall that $O_{3}=(\pi, 0)$ in $(\alpha, \beta)$ coordinates is the unique intersection point of the singular manifolds $\omega_{1}, \omega_{2}$. The procedure is similar to that used in section 4 for $O_{i}=1,2$, namely a factorization of the Hamiltonian (2.6) is carried out in a sufficiently small ball of $O_{3}$ after a suitable change of coordinates. Like the previous cases, the leading singular term or unperturbed Hamiltonian represents decoupled oscillators with two degrees of freedom.

To begin, we introduce new coordinates with origin located at $\mathrm{O}_{3}$. Since there is little possibility of confusion these new coordinates are also denoted by $(a, b)$,

$$
a=\alpha-\pi, \quad b=\beta .
$$

Substituting (5.1) in (2.6) and expanding the trigonometric terms $\sin a, \sin b, \cos a$ and $\cos b$, we obtain (after absorbing a constant multiple in the time) the Hamiltonian

$$
H(a, b ; G)=\log \left[\frac{1+\cos G}{a^{2}-b^{2}}\right]+\log \{K(a, b ; G)\}
$$

The singular first term is

$$
H_{0}(a, b)=-\log \left[a^{2}-b^{2}\right]+\log [1+\cos G] .
$$

Henceforth the constant second term in (5.3) parametrized by $G$ will be dropped since the Hamiltonians are defined up to an additive constant. The second term in (5.2) is

$$
H_{1}(a, b ; G)=\log [1+\text { h.o.t. }] \text {. }
$$

$K(a, b ; G)$ is a convergent power series in $a, b$ with coefficients depending smoothly on $G$ and begins with the term one. Thus in a sufficiently small ball around the origin of the coordinate system (5.1), the Hamiltonian $H(a, b ; G)$ is a small perturbation of $H_{0}(a, b)$.

The Hamilton's equations for (5.3),

$$
\frac{\mathrm{d} \bar{a}}{\mathrm{~d} \tau}=\frac{2 \mathrm{i} b}{a^{2}-b^{2}}=\frac{\mathrm{i} \partial H_{0}}{\partial b}, \quad \frac{\mathrm{d} \bar{b}}{\mathrm{~d} \tau}=\frac{2 \mathrm{i} a}{a^{2}-b^{2}}=\frac{\mathrm{i} \partial H_{0}}{\partial a},
$$

can be diagonalized or decoupled by a second coordinate transformation,

$$
A=a+b, \quad B=b-a .
$$

In these variables, the Hamiltonian (5.2) becomes

$$
\begin{aligned}
& H(A, B ; G)=H_{0}(A, B)+H_{1}(A, B ; G), \\
& H_{0}(A, B)=-\log (A B), \\
& H_{1}(A, B)=\log [K(A, B ; G)],
\end{aligned}
$$

where $K(A, B ; G)$ is again a convergent power series in $A, B$ with first term equal to one. The 
Hamiltonian's equations for $H_{0}(A, B)$ are

$$
\frac{\mathrm{d} \bar{A}}{\mathrm{~d} \tau}=\frac{-\mathrm{i}}{A}=\mathrm{i} \frac{\partial H_{0}}{\partial A}, \quad \frac{\mathrm{d} \bar{B}}{\mathrm{~d} \tau}=\frac{\mathrm{i}}{B}=-\mathrm{i} \frac{\partial H_{0}}{\partial B},
$$

which are clearly decoupled oscillators equations.

Once again the coordinates (5.6) which decouple the equations are natural in the sense that they give the trivial embedding of the singular manifolds $\omega_{1}, \omega_{2}$ which intersect at $O_{3}$, that is

$$
\begin{aligned}
& \omega_{1}=\{(A, B) \mid A \in \mathbb{C}, B=0\}, \\
& \omega_{2}=\{(A, B) \mid A=0, B \in \mathbb{C}\} .
\end{aligned}
$$

Next, we introduce action-angle variables

$$
A=\sqrt{J_{1}} \mathrm{e}^{\mathrm{i} \theta_{1}}, \quad B=\sqrt{J_{2}} \mathrm{e}^{\mathrm{i} \theta_{2}}
$$

The full Hamiltonian becomes

$$
H\left(J_{1}, J_{2}, \theta_{1}, \theta_{2} ; G\right)=H_{0}\left(J_{1}, J_{2}\right)+H_{1}\left(J_{1}, J_{2}, \theta_{1}, \theta_{2} ; G\right),
$$

where the leading term

$$
H_{0}\left(J_{1}, J_{2}\right)=-\log \left(J_{1} J_{2}\right)
$$

is independent of $\theta_{1}, \theta_{2}$. The perturbation $H_{1}$ has the real analytic form

$$
H_{1}\left(J_{1}, J_{2}, \theta_{1}, \theta_{2} ; G\right)=\log \left[K\left(J_{1}, J_{2}, \theta_{1}, \theta_{2} ; G\right)\right]=\log [|K(A, B ; G)|] .
$$

Since $K\left(J_{1}, J_{2}, \theta_{1}, \theta_{2} ; G\right)$ is the modulus of $K(A, B ; G)$ in (5.7), it is a convergent power series in $J_{1}, J_{2}$ and the sines and cosines of $\theta_{1}, \theta_{2}$, with coefficients which depend smoothly on $G$. Moreover the power series begins with the term one.

The frequencies of the $S^{1} \times S^{1}$ orbits of the leading equations

$$
\begin{array}{ll}
\frac{\mathrm{d} J_{1}}{\mathrm{~d} \tau}=0, & \frac{\mathrm{d} \theta_{1}}{\mathrm{~d} \tau}=\frac{\partial H_{0}\left(J_{1}, J_{2}\right)}{\partial J_{1}}=-\frac{1}{J_{1}}, \\
\frac{\mathrm{d} J_{2}}{\mathrm{~d} \tau}=0, & \frac{\mathrm{d} \theta_{2}}{\mathrm{~d} \tau}=\frac{\partial H_{0}\left(J_{1}, J_{2}\right)}{\partial J_{2}}=-\frac{1}{J_{2}}
\end{array}
$$

depend on the values of the action variables.

We are ready to state and prove an analogue of theorem 4.5 for a neighborhood of the singular intersection point $\mathrm{O}_{3}$.

Theorem 5.1. In a sufficiently small open ball $S_{\mathfrak{\varepsilon}}, \varepsilon(G) \ll 1$ around the point $O_{3}$ (the origin), the full Hamiltonian (5.11) admits a set of quasi-periodic solutions which has positive Lebesgue measure in $\mathbb{R}^{4}$. 
Proof. First we check that the unperturbed Hamiltonian $H_{0}\left(J_{1}, J_{2}\right)$ is nondegenerate for all values of $J_{1}, J_{2}$. The determinant of the Hessian matrix

$$
\left|\frac{\partial^{2} H_{0}}{\partial J_{l} \partial J_{k}}\right|=\frac{\partial^{2} H_{0}}{\partial J_{1}^{2}} \frac{\partial^{2} H_{0}}{\partial J_{2}^{2}}=\frac{1}{J_{1}^{2} J_{2}^{2}} \neq 0 .
$$

Second, the full Hamiltonian $H\left(J_{1}, J_{2}, \theta_{1}, \theta_{2} ; G\right)$ is real analytic in $J_{1}, J_{2}, \theta_{1}, \theta_{2}$ and $2 \pi$-periodic in $\theta_{1}, \theta_{2}$ in $S_{\varepsilon}$. Moreover for $\varepsilon(G)$ sufficiently small, the full Hamiltonian is a small $(\mathcal{O}(\varepsilon)$ ) perturbation of the integrable leading term, $H_{0}\left(J_{1}, J_{2}\right)$, (cf. (5.13)). Thus by the KAM theorem, the Hamiltonian (5.11) admits a positive measure set of tori which are continuations of the sufficiently irrational tori of $H_{0}\left(J_{1}, J_{2}\right)$.

We remark that theorem 5.1 differs from theorem 4.6 in the sense that the existence of quasi-periodic solutions is established in a full neighborhood, that is an open ball $S_{\varepsilon}$ of the point $O_{3}$. On the other hand, the quasi-periodic solutions or KAM tori in theorem 4.5 are found in the restricted subsets of $S_{\varepsilon}^{i}$ denoted by $M_{\delta, n}$ (see (4.10)) which are constructed to be nearer to the singular manifold $\omega_{i}$ than to either $\mu_{i}$ or $\nu_{i}$, $i=1,2$. (Recall that the points of intersection $O_{i}, i=1,2$ are phase points where the three singular manifolds meet.) The singular manifolds $\mu_{i}, \nu_{i}$ correspond to merging of opposite vortex rows and we should expect unbounded solutions rather than KAM tori in their vicinity. Like its counterpart, theorem 5.1 holds for all values of the complex parameter $G$. Thus in each $C$-level manifold of the eqs. (A.1), there is a positive measure set of tori in the neighborhood of $\mathrm{O}_{3}$.

\section{Open connected sets of bounded solutions}

In this section, we prove several useful corollaries of the results in sections 4 and 5. Collectively, theorem 4.5, theorem 4.6 and theorem 5.1 establish the existence of relatively large (in the sense of measure) sets of invariant tori in the neighborhoods of $O_{i}, i=1,2,3$ in each $C$-level manifold of the eqs. (A.1). The first idea here is to show that in fact there are open connected sets of bounded solutions near these singular intersection points. The second idea is to consider the collection of bounded solutions parametrized smoothly by $G$ in the 6-dimensional phase space of the equation for four infinite rows, (A.1).

The key property used in the proof of the following results is the fact that in 4-dimensional phase space, the KAM tori separate an isoenergetic manifold into two distinct regions (cf. Arnol'd [23]).

First we construct bounded, open and connected sets that are also invariant regions for the Hamiltonian system in the neighborhood of the intersection points $O_{i}, i=1,2$. Let $S_{i}$ denote $\varepsilon$-radius balls around $O_{i}$. For $\varepsilon$ sufficiently small, theorem 4.6 implies that there are large sets of invariant tori in $S_{i}$, parametrized over a Cantor set of strongly nonresonant frequencies, $\left(\omega_{1}, \omega_{2}\right)$, for which there exist $C$ and $\nu$ such that $\left|\omega_{1} k_{1}+\omega_{2} k_{2}\right|>C|k|^{-\nu}$ for all integral 2-vectors $k=\left(k_{1}, k_{2}\right)$. These tori depend continuously on $\left(\omega_{1}, \omega_{2}\right)$ and their rotation numbers, $\omega$, are given by $\omega_{1} / \omega_{2}$. Through each point $\omega$ in the Cantor set, consider the short segment

$$
\left(t \omega_{1}, t \omega_{2}\right), \quad 1-\delta<t<1+\delta,
$$

with constant rotation number $\omega=\omega_{1} / \omega_{2}$. This one-parameter family satisfies the strongly nonresonant condition for sufficiently small $\delta$ which depends on $\left(\omega_{1}, \omega_{2}\right)$ and $C, \nu$. To each such family there is a 
continuous curve of invariant tori $T^{i}(t)$. The union of their interiors, $T^{i}$, is an invariant connected and bounded set in 4-dimensional phase space which contains the singular point $\left(J_{1}, J_{2}\right)=(0,0)$.

Theorem 6.1. For each fixed value of $G$, the Hamiltonian $H(\alpha, \beta ; G)(2.6)$ admits bounded connected and invariant sets in the neighborhood of the intersection points $O_{i}, i=1,2$. Moreover each of these invariant sets is an open subset in $\mathbb{R}^{4}$.

Proof. The constructed sets $T^{i}, i=1,2$, for some fixed rotation number $\omega$ are bounded and connected. They are also invariant sets because the interiors of the tori $T^{i}(t)$ in the 3-dimensional isoenergetic manifold $M_{h(t)}$ are invariant. These sets are also open in $\mathbb{R}^{4}$ because the one-parameter family $T^{i}(t)$ is not contained in a single $h$-level surface $M_{h(t)}$, i.e. $h(t)$ is not a constant. If the family of tori is in a single $h$-level surface then one obtains a contradiction with the isoenergetic nondegeneracy condition which holds for the unperturbed Hamiltonian $H_{0}$ in (4.12), as seen in the following:

$$
\left|\begin{array}{ccc}
\frac{\partial^{2} H_{0}}{\partial J_{1}^{2}} & \frac{\partial^{2} H_{0}}{\partial J_{1} \partial J_{2}} & \frac{\partial H_{0}}{\partial J_{1}} \\
\frac{\partial^{2} H_{0}}{\partial J_{1} \partial J_{2}} & \frac{\partial^{2} H_{0}}{\partial J_{2}^{2}} & \frac{\partial H_{0}}{\partial J_{2}} \\
\frac{\partial H_{0}}{\partial J_{1}} & \frac{\partial H_{0}}{\partial J_{2}} & 0
\end{array}\right|=\left|\begin{array}{ccc}
-\frac{1}{J_{1}^{2}} & 0 & -\frac{1}{J_{1}} \\
0 & -\frac{2}{J_{2}^{2}} & \frac{2}{J_{2}} \\
-\frac{1}{J_{1}} & \frac{2}{J_{2}} & 0
\end{array}\right|=\frac{2}{J_{1}^{2} J_{2}^{2}}-\frac{4}{J_{1}^{2} J_{2}^{2}}=-\frac{2}{J_{1}^{2} J_{2}^{2}} .
$$

Thus the constructed sets $T^{i}, i=1,2$, are not contained in a single energy manifold and the theorem is proven.

Next, we construct a bounded connected and invariant open set $T^{3}$ in the neighborhood of the intersection point $\mathrm{O}_{3}$. The construction follows somewhat different steps because the boundedness requirement for $T^{3}$ implies that we cannot use the full interior of the invariant tori $T^{3}(t)$ in the energy manifold $M_{h(t)}$. In the coordinates (5.10) in $\varepsilon$-radius ball $S_{3}$ around $O_{3}$ is given by

$$
S_{3}=\left\{\left(J_{1}, J_{2}, \theta_{1}, \theta_{2}\right) \mid+J_{1}+J_{2} \leq \varepsilon\right\} .
$$

For $\varepsilon$ sufficiently small, the Hamiltonian (5.7) restricted to $S_{3}$ is greater than some large positive number, i.e. $H=h>N$. The Hamiltonian is essentially given by its leading term $H=-\log \left(J_{1}, J_{2}\right)(5.12)$ in $S_{3}$ which satisfies the isoenergetic nondegeneracy condition of the KAM theorem,

$$
\left|\begin{array}{ccc}
\frac{\partial^{2} H_{0}}{\partial J_{1}^{2}} & \frac{\partial^{2} H_{0}}{\partial J_{1} \partial J_{2}} & \frac{\partial H_{0}}{\partial J_{1}} \\
\frac{\partial^{2} H_{0}}{\partial J_{1} \partial J_{2}} & \frac{\partial^{2} H_{0}}{\partial J_{2}^{2}} & \frac{\partial H_{0}}{\partial J_{2}} \\
\frac{\partial H_{0}}{\partial J_{1}} & \frac{\pi H_{0}}{\partial J_{2}} & 0
\end{array}\right|=\left|\begin{array}{ccc}
-\frac{1}{J_{1}^{2}} & 0 & -\frac{1}{J_{1}} \\
0 & -\frac{1}{J_{2}^{2}} & \frac{1}{J_{2}} \\
-\frac{1}{J_{1}} & \frac{1}{J_{2}} & 0
\end{array}\right|=\frac{1}{J_{1}^{2}}\left(-\frac{1}{J_{2}^{2}}\right)-\left(\frac{1}{J_{1}}\right)\left(\frac{1}{J_{1} J_{2}^{2}}\right)=-\frac{2}{J_{1}^{2} J_{2}^{2}}
$$


Thus there is a large set of invariant tori on each isoenergetic surface $M_{h} \cap S_{3}$. Each such torus is given by a smooth function

$$
J_{1}=f_{\omega}\left(\theta_{1}, \theta_{2} ; h\right)
$$

in the 3-dimensional surface $M_{h} \cap S_{3}$ where the index $\omega$ is the rotation number $\omega_{1} / \omega_{2}$. Note that (6.5) gives a natural ordering of the tori $T_{\omega}^{3}(h)$ in $M_{h}$, i.e. $T_{\omega}^{3}(h)$ is inside $T_{\omega^{\prime}}^{3}$, if $f_{\omega}<f_{\omega^{\prime}}$ and $\omega<\omega^{\prime}$ (using the fact that a torus separates $M_{h}$ into inside and outside regions). Now it is clear from the forms of $H_{0}(5.12)$ and $S_{3}(6.3)$ that the full interior of a torus $T_{\omega}^{3}(h)$ in $M_{h} \cap S_{3}$ is not contained in $S_{3}$.

From the above considerations, it is therefore necessary to choose two tori $T_{\omega}^{3}(h)$ and $T_{\omega^{\prime}}^{3}(h)$ such that the one with rotation number $\omega$ is inside the other and both are in $M_{h} \cap S_{3}$.

Thus the annular region

$$
T_{\omega^{\prime}}^{3}(h) \backslash T_{\omega}^{3}(h)=A(h)
$$

is contained in $S_{3}$. The remaining steps in the construction follow closely the previous ones for theorem 6.1. Theorem 5.1 implies that there is a large set of invariant tori in $S_{3}$ parametrized over a Cantor set of strongly nonresonant frequencies $\left(\omega_{1}, \omega_{2}\right)$. Through the points $\omega<\omega^{\prime}$, there are two short segments

$$
\begin{array}{ll}
\left(t \omega_{1}, t \omega_{2}\right), & 1-\delta<t<1+\delta \\
\left(t \omega_{1}^{\prime}, t \omega_{2}^{\prime}\right), & 1-\delta^{\prime}<t<1+\delta^{\prime}
\end{array}
$$

each has constant rotation numbers

$$
\omega=\omega_{1} / \omega_{2}, \quad \omega^{\prime}=\omega_{1}^{\prime} / \omega_{2}^{\prime}
$$

We choose $\left(\omega_{1}, \omega_{2}\right)$ and $\left(\omega_{1}^{\prime}, \omega_{2}^{\prime}\right)$ so that the corresponding tori for $t=1$ are in the $h$-level surface $M_{h} \cap S_{3}$. Let $\delta_{\mathrm{m}}=\min \left\{\delta, \delta^{\prime}\right)$ and consider the continuous one-parameter family of annular regions,

$$
A(t)=T_{\omega^{\prime}}^{3}(h(t)) \backslash T_{\omega^{\prime}}^{3}(h(t)), \quad 1-\delta_{\mathrm{m}}<t<1+\delta_{\mathrm{m}} .
$$

It is easy to verify that for the above values of $t, T_{\omega}^{3}(h(t))$ and $T_{\omega^{\prime}}^{3}(h(t))$ are in the same isoenergetic manifold $M_{h(t)} \cap S_{3}$. We take the union to be our set $T^{3}$

$$
T^{3}=\bigcup_{t} A(t)
$$

Theorem 6.2. For each fixed value of $G$, the Hamiltonian (2.6) admits a bounded invariant connected set in the neighborhood of $O_{3}$. Moreover this set is open in $\mathbb{R}^{4}$.

Proof. From the construction of $T^{3}$ it is clear that the union is an invariant connected set since the annular regions $A(t)$ are invariant. Each $A(t)$ is contained in $S_{3}$ and therefore the union is bounded. The openness of $T^{3}$ follows from the same argument for the transversality of the continuous one-parameter family $A(t)$ to each $h$-level surface $M_{h(t)}$, as in the proof of theorem 6.1. The sufficient condition for transversality is the isoenergetic nondegeneracy condition (6.4).

Next we discuss the smooth dependence of the open invariant sets, $T^{i}, i=1,2,3$, on the parameter $G$. Since the $C$-level manifolds are continuously embedded in the 6-dimensional phase space of (A.1) as $G$ 
varies, and the positions of the singular manifolds $\mu_{i}, \nu_{i}, \omega_{i}, i=1,2$, within the $C$-level sets change continuously with $G$, we deduce that the above invariant sets vary continuously with $G$.

Consider the union (over values of $G$ in a small open complex disk $D$ ) of the invariant sets $T^{i}(G)$, $i=1,2,3$,

$$
V_{i}=\bigcup_{G \in D} T^{i}(G)
$$

Then $V_{i}, i=1,2,3$, are bounded, open, connected invariant subsets in $\mathbb{R}^{6}$ for the eqs. (A.1). These sets are constructed near the corresponding singular intersection points $O_{i}(G), i=1,2,3$.

The openness property of the sets $V_{i}$ will be used to establish the persistence of certain vortex patterns under small perturbations. In other words the topological property of openness is equivalent to the physical property of being capable of observation.

\section{Generalizations and conclusions}

In the first part of this section we discuss the physical consequences of the mathematical results in this paper. Generalizations of our procedure to other classes of Hamiltonians which admit complex singularities are outlined in the second part.

We recall the physical meaning of the singular manifolds $\mu_{i}, \nu_{i}, \omega_{i}, i=1,2$, for the Hamiltonian (2.6) from section 3 . Also recall the definitions of a vortex pair, vortex couple and a vortex triplet from the same section.

Theorem 6.1 implies that if three of the four vortex rows are clustered together and in addition, two such rows of similar circulation are sufficiently near each other, then this configuration is preserved for all time. In terms of the above definitions, this vortex configuration is a vortex pair within a vortex triplet. The remarks concerning the smooth dependence on the parameter $G$ of the invariant sets $T_{i}, i=1,2,3$, implies moreover that given any such vortex configuration, sufficiently small perturbations from it will evolve into vortex states similar to the original configuration.

The above statement on the persistence of the vortex pair within a vortex triplet configuration holds only for perturbations in the 6-dimensional phase space of the equations for four vortex rows (A.1). Such configurations are special solutions of the vortex lattices (see fig. 1), namely spatially periodic solutions with the shortest wavelength. Since the solution space of the vortex lattices are infinite dimensional and consists of periodic signals of all wavelengths, we do not claim that the special configuration discussed above will persist under arbitrary small perturbations of the vortex lattices.

Theorem 6.2 implies an analogue statement for the vortex configuration consisting of two distinct vortex pairs. If the separations between the vortex rows within each pair are sufficiently small compared to the distance between the two vortex pairs, then the configuration will be preserved for all time. Moreover the smooth dependence on the parameter $G$ implies that sufficiently small perturbations (within the phase space of (A.1)) of this configuration evolve into vortex states with similar combinatorial configuration.

In addition, theorems 4.5, 4.6 and 5.1 imply that a substantial fraction (of positive Lebesgue measure in $\mathbb{R}^{6}$ ) of vortex states with one of the above special configurations evolve in a quasi-periodic manner. They represent two-frequencies oscillations of vortex triples and vortex pairs. The comparison of these theoretical results with the experimental observations of vortex merging in the wake of an oscillating cylinder as reported in Williamson and Roshko [22] and Couder [23], is given in Lim and Sirovich [14]. In the above paper we also report numerical studies of eqs. (A.1) which indicated the presence of quasi-periodic motions in vortex lattices and have guided the investigations in the present paper. 


\subsection{Generalizations}

Now, we wish to indicate briefly some applications of the method illustrated in this paper to more general situations. An immediate generalization is to consider arbitrary $N$-periodic motions in vortex lattices by studying the singular manifolds of eqs. (2.2) for $N>2$. Other applications arise in problems of $N$ oscillators in a ring which interact through potentials besides the logarithm function.

In general, the singular hypersurfaces of the Hamiltonians are not embedded submanifolds in phase space. For our method to work, the singular surfaces must intersect locally like submanifolds. Other necessary and sufficient conditions for the method of singular manifolds will be discussed in another paper.

We close with the remark that the theoretically significant example (2.6) analyzed in this paper can be discussed within the abstract framework in Marsden and Weinstein [24]; but we choose to use the more familiar language in this paper in order to highlight the simple geometrical ideas of our method.

\section{Acknowledgements}

The author would like to thank Professor L. Sirovich for introducing him to vortex dynamics. He would like to thank one of the referees for suggesting improvements in section 6 . He would also like to acknowledge the professional typing of this manuscript by Dee Hudock.

\section{Appendix}

Consider the case $N=2$ for eqs. (2.2),

$$
\frac{\mathrm{d} \bar{Z}_{k}}{\mathrm{~d} t}=\sum_{j=1}^{4}(-1)^{j} \frac{\Gamma}{4 l \mathrm{i}} \cot \frac{\pi}{2 l}\left(Z_{k}-Z_{j}\right), \quad k=1,2,3,4 .
$$

Writing in terms of the differences $\left(Z_{1}-Z_{2}\right),\left(Z_{2}-Z_{3}\right)$ and $\left(Z_{3}-Z_{4}\right)$, eqs. (A.0) become (where only the equation for $\left(Z_{1}-Z_{2}\right)$ is given explicitly)

$$
\begin{aligned}
\frac{\mathrm{d}\left(\overline{Z_{1}-Z_{2}}\right)}{\mathrm{d} t}= & \frac{\Gamma}{4 l \mathrm{i}} \cot \frac{\pi}{2 l}\left[\left(Z_{1}-Z_{2}\right)+\left(Z_{2}-Z_{3}\right)+\left(Z_{3}-Z_{4}\right)\right] \\
& +\frac{\Gamma}{4 l \mathrm{i}} \cot \frac{\pi}{2 l}\left[\left(Z_{1}-Z_{3}\right)\right]-\frac{\Gamma}{4 l \mathrm{i}} \cot \frac{\pi}{2 l}\left[\left(Z_{1}-Z_{2}\right)+\left(Z_{2}-Z_{3}\right)\right] \\
& -\frac{\Gamma}{4 l \mathrm{i}} \cot \frac{\pi}{2 l}\left[\left(Z_{2}-Z_{3}\right)+\left(Z_{3}-Z_{4}\right)\right], \\
\frac{\mathrm{d}\left(\overline{Z_{2}-Z_{3}}\right)}{\mathrm{d} t}= & \cdots, \\
\frac{\mathrm{d}\left(\overline{Z_{3}-Z_{4}}\right)}{\mathrm{d} t}= & \cdots .
\end{aligned}
$$

The equations which are not given explicitly can be obtained directly from (A.0) and are left to the reader.

It can be verified directly from (A.1), which governs the interaction of four vortex rows, that the complex function

$$
C=\left(Z_{1}-Z_{2}\right)+\left(Z_{3}-Z_{4}\right)
$$


is an invariant. We consider the reduced flow of (A.1) on the level sets of $C$, that is we eliminate the difference $\left(Z_{3}-Z_{4}\right)$ with the help of (A.2).

Following Kochin [13], we give the reduction of (A.1) in terms of the normalized perturbations which are given by

$$
Z_{k}=V t+Z_{k 0}+\frac{2 l}{\pi} \rho_{k}, \quad k=1,2,3,4,
$$

where $Z_{k 0}$ gives the equilibrium configuration (see fig. 1) and $V$ is the rigid translation velocity of the equilibrium.

Each value of the invariant $C$ specifies a particular equilibrium vortex trail, i.e.

$$
\begin{aligned}
C & =\left(Z_{10}-Z_{20}\right)+\left(Z_{30}-Z_{40}\right) \\
& =2(b+\mathrm{i} h),
\end{aligned}
$$

where $b$ is the stagger and $h$ is the separation between the two vortex rows in equilibrium (cf. fig. 1).

In terms of the normalized differences

$$
\alpha=2\left(\rho_{2}-\rho_{1}\right), \quad \beta=\left(\rho_{2}-\rho_{3}\right),
$$

the reduced flow of (A.1) on the $C$-level manifolds is given by

$$
\begin{aligned}
& \frac{\mathrm{d} \bar{\alpha}}{\mathrm{d} \tau}=-\sin \beta\left[\frac{1}{\cos \alpha+\cos \beta}-\frac{1}{\cos \beta+\cos G}\right], \\
& \frac{\mathrm{d} \bar{\beta}}{\mathrm{d} \tau}=-\sin \alpha\left[\frac{1}{\cos \alpha+\cos \beta}-\frac{1}{\cos \beta+\cos G}\right] .
\end{aligned}
$$

The complex parameter $G$ given by

$$
G=\frac{\pi}{2 l} C
$$

specifies the particular $C$-level manifold. Time in eqs. (A.6) has been scaled in the following way:

$$
\tau=\Gamma \pi t / 2 l^{2}
$$

\section{References}

[1] K.R. Meyer, J. Diff. Eq. 39 (1981) 2.

[2] M. Braun, J. Diff. Eq. 13 (1973) 300.

[3] M. Kummer, Commun. Math. Phys. 48 (1976) 53.

[4] M. Kummer, Commun. Math. Phys. 58 (1978) 85.

[5] R.C. Churchill, M. Kummer and D.L. Rod, J. Diff. Eq. 49 (1983) 359.

[6] F.G. Gustavson, Astron. J. 71 (1966) 670.

[7] J.M. Greene, Physica 18D (1986) 427.

[8] G. Contopoulos, Astrophys. J. 138 (1963) 1297.

[9] T. von Karman, Gott. Nach. Math. Phys. K1. (1911) 509. 
[10] L. Sirovich, Phys. Fluids 28 (1985) 2723.

[11] K.R. Sreenivasan, in: Frontier in Fluid Mechanics, S.H. Davis and J.L. Lumley, eds. (Springer, New York, 1985 ), p. 41.

[12a] C.C. Lim and L. Sirovich, Phys. Fluids 29 (1986) 3910.

[12b] C.C. Lim and L. Sirovich, Bounded and unbounded solutions of the von Karman vortex trail, submitted to SIAM J. Appl. Math.

[13] N.E. Kochin et al., Theoretical Hydromechanics (Interscience, New York, 1964).

[14] C.C. Lim and L. Sirovich, Nonlinear vortex trail dynamics, to appear in Phys. Fluids.

[15] J.L. Synge, Can. J. Math. 1 (1949) 257.

[16] C.C. Lin, Proc. Natl. Acad. Sci. USA 27 (1941) 570.

[17] E.A. Novikov, Ann. N.Y. Acad. Sci. 357 (1980) 47.

[18] H. Aref, Phys. Fluids 22 (1979) 393.

[19] E.A. Novikov and Yu.B. Sedov, Sov. Phys. JETP 48 (1978) 440.

[20] K.M. Khanin, Physica 4D (1982) 261.

[21] C.C. Lim, On singular manifolds, integrals and weakly coupled oscillators in Hamiltonians, in preparation.

[22] V.I. Arnol'd, Mathematical Methods of Classical Mechanics (Springer, Berlin, 1980).

[23] V.I. Arnol'd, Russ. Math. Surv. 18 (5) (1963) 9.

[24] J. Moser, Stable and Random Motions in Dynamical Systems (Princeton University Press, Princeton, 1973).

[25] J. Moser, Nachr. Akad. Wiss. Gott. Math. Phys. Kl. 2 (1982) 1.

[26] C.H.K. Williamson and A. Roshko, Vortex dynamics in the wake of an oscillating cylinder, Bull. Am. Phys. Soc. 31 (1986) 1690.

[27] Y, Couder et al., in: Lecture Notes in Physics, vol. 23 (Springer, Berlin, 1984), p. 327.

[28] J. Marsden and A. Weinstein, Physica 7D (1983) 305. 